\title{
Linear instability analysis of thermocapillary convection in a bilayer system
}

\author{
Zijing Ding ${ }^{\mathrm{a}, \mathrm{b}}$, Qiusheng Liu ${ }^{\mathrm{a}, *}$ \\ ${ }^{a}$ Key Laboratory of Microgravity (National Microgravity Laboratory), Institute of Mechanics, Chinese Academy of Sciences, Beijing 100190, China \\ ${ }^{\mathrm{b}}$ Thermal and Fluid Division, School of Mechanical and Aerospace, Nanyang Technological University, 639798 Singapore, Singapore
}

\section{A R T I C L E I N F O}

Article history:

Received 28 April 2012

Received in revised form 12 November 2012

Accepted 26 February 2013

Available online 20 March 2013

\section{Keywords:}

Two-layer flow system

Linear stability analysis

Thermocapillary convection

\begin{abstract}
A B S T R A C T
The linear instability analysis of thermocapillary convection in a bilayer system consisting of silicon oil 10cS and fluorinert FC70 liquids was discussed in this paper. The bilayer system was bound below by a rigid plate and above by a free surface with a passive gas. The two immiscible liquids were separated by an interface. A constant horizontal temperature gradient was imposed along the interfaces. Two typical cases were studied: (i) streamwise homogeneous disturbances (the streamwise disturbance wave number $\alpha=0$ ); (ii) spanwise homogeneous disturbances (the spanwise disturbance wave number $\beta=0$ ). When $\alpha=0$, it was found that convection in the two layers may occur in the form of stationary mode or oscillatory mode. The oscillatory mode takes the form of traveling wave, which propagates in either spanwise direction. When $\beta=0$, convection in the two layers occurs in the form of oscillatory mode, which takes the form of traveling wave propagating in the same direction as base flow. The three dimensional analysis suggested that disturbance in the spanwise direction was the major cause of destabilization in the system. The influences of Biot number $B i$ and the depth ratio $h$ on the unstable modes in the bilayer system were discussed.
\end{abstract}

(c) 2013 Elsevier Ltd. All rights reserved.

\section{Introduction}

The phenomenon of thermocapillary instability has received much attention in recent years. Colinet et al. [1] and Joseph and Renardy [2] investigated the stability and pattern formation of heat convection problems. Pearson [3] investigated the classical Marangoni convection in a single liquid layer heated from below. Linear stability analysis of Pearson predicts the threshold value of Marangoni number, $M a=79.6$, which coincides with experiments. Smith and Davis [4,5] and Davis [6] considered thermocapillary instabilities in a single layer system with a non-deformable interface. Sen and Davis [7] studied steady thermocapillary flows in two-dimensional slots. In [4-7], the flow system was subjected to a constant horizontal temperature gradient along the interface resulted in a linearly distributed horizontal flow, i.e. the thermal Couette flow. Particularly, Smith and Davis [4] found that for this linear base flow, when it was subjected to spanwise disturbances, convection may occur in the form of stationary longitudinal rolls, or in the form of hydrothermal waves. The hydrothermal wave only occurs when the Prandtl number is small.

Zeren and Reynolds [8] analytically and experimentally discussed thermal convection instabilities in a two-fluid layer system which was heated from below or above. Wahal and Bose [9] considered a bilayer system with a motionless basic state which was

\footnotetext{
* Corresponding author.

E-mail addresses: zding001@e.ntu.edu.sg (Z. Ding), liu@imech.ac.cn (Q. Liu).
}

heated from below. Interaction between buoyancy and interfacial modes and the oscillatory mode were discussed. Rasenat et al. [10] investigated the Rayleigh-Bénard convection in a bilayer system consisting of two immiscible fluids which were bound by two parallel rigid plates and separated by a deformable interface. Stationary mode and oscillatory mode were discussed in their paper. Liu and Roux [11] and Liu et al. [12] extended the problem to Rayleigh-Bénard-Marangoni convection in a bilayer system by the linear stability analysis. Liu et al. predicted that when the depth ratio (lower layer's thickness/upper layer's thickness) was bound in some region, the oscillating convection in the bilayer system may be observed. Li et al. [13] studied the two-dimensional base state of an annular two-layer pool. The system was heated from outer cylindrical wall and cooled at the inner cylindrical wall. Both asymptotic method and direct numerical simulation were carried out by Li et al. to study the steady laminar two-dimensional thermocapillary flow which showed the results by asymptotic method and numerical experiment were in agreement. However, the stability of flow system was not investigated.

Numerical study of thermocapillary flows in a three-layer system subjected to a constant temperature gradient along the interfaces was carried out by Simanovskii [14]. Simanovskii et al. [15] further investigated anticonvection and Rayleigh-Bénard convection in two-layer systems by numerical study. In [14,15], direct simulation preformed the nonlinear dynamics of system, and the finite-difference method was applied by considering a periodic boundary conditions in the lateral direction. Nepomnyashchy and 


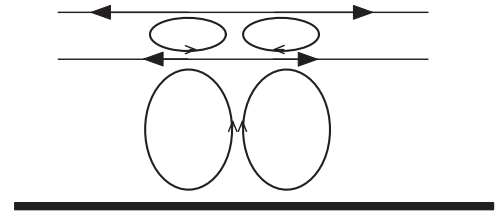

(a)

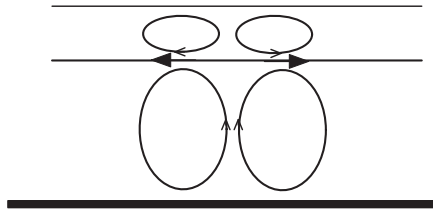

(b)

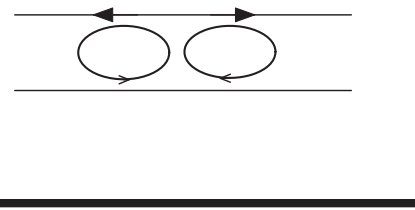

(c)

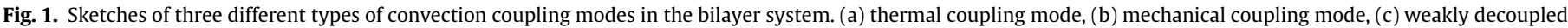
mode.

Simanovskii [16] studied Marangoni instability in ultrathin twolayer films, and van der Waals forces were included in their model. Both linear and nonlinear analyses were employed to investigate the problem [16]. Nepomnyashchy and Simanovskii [17] further investigated dynamics of the two-layer films subjected to horizontal temperature gradient under the gravity effect. Long-wave theory was applied to study the linear stability of the system and the nonlinear evolution of the interfaces.

Recently, Nepomnyashchy and Simanovskii [18] reported nonlinear Marangoni waves in a heated two-layer film in the presence of gravity by a lubrication approximation. Both linear stability analysis and three dimensional nonlinear evolution study were carried out. The linear stability analysis revealed the existence of stationary and oscillatory unstable modes in this bilayer system. However, the complex coupling modes in the two-layer flow system could not be studied by the long-wave theory [16-18]. Aside from that, to our knowledge, in previous works, oscillatory modes in multi-layer flow systems are all coupled with Rayleigh effects.

In this paper, we investigated the thermocapillary convection in a bilayer system to study all the possible coupling modes without buoyancy effects, using numerical methods. The interfaces were assumed non-deformable. First, it is helpful to discuss the physical mechanisms of possible unstable modes in the bilayer system as shown in Fig. 1. In this bilayer system, there are two main forces that can drive thermal convection. One is the gas-liquid interface tension, and the other is the liquid-liquid interface tension. The type of convection may change if the depth ratio (upper layer's thickness/lower layer's thickness) between the two layers changes. In Fig. 1(a), when the depth ratio is small, variation of either surface tension due to random disturbances may initiate co-rotating rolls in the bilayer system. This unstable mode is called thermalcoupling mode. Physically, suppose there exists a hot spot on the liquid-liquid interface, and due to the small depth of the upper layer, a temperature gradient would be generated at gas-liquid interface through heat conduction resulted in co-rotating rolls in the upper layer. If convection would not be driven by the gasliquid interface tension because of the cooling of bounding gas, and would only be driven by the liquid-liquid interface tension, then counter-rotating rolls can be seen as shown in Fig. 1(b). In this paper, this type of convection is called mechanical coupling mode. As increasing the upper layer's depth, convection may not occur in the lower layer as shown in Fig. 1(c), and this type of convection is called weakly decoupled mode in this paper. All of the three possible modes occur in the form of stationary rolls, while the interactions between these modes may initiate oscillatory mode. We will discuss these modes in the following sections.

This paper is organized as follows; the paper begins with mathematical formulation of the problem in Section 2; in which, base state of the flow system and the linearized perturbed system are given. In Sections 3 and 4, results are presented, including two typical cases: the streamwise homogeneous disturbances case $\alpha=0$ and spanwise homogeneous disturbances case $\beta=0$. The influences of depth ratio and Biot number on the nature of convection are discussed. In Section 5, the three-dimensional perturbed problem is investigated to study the most preferred unstable mode. A conclusion remark is made in the end.

\section{Mathematical model}

The sketch of the bilayer system is shown in Fig. 2. A constant horizontal temperature gradient $\partial T / \partial x=-b, b>0$, is imposed along the system. The upper fluid is silicon oil 10cS, and the lower is fluorinert FC70. The physical properties of the two liquids are listed in Table 1 . Both the two fluids are considered Newtonian. The surface tension of many liquids can be considered as linear function of temperature: $\sigma=\sigma_{0}-\gamma\left(T-T_{0}\right)$, where $T_{0}$ is the reference temperature, $\gamma=-\partial_{T} \sigma$. In this paper, the gas-liquid and liquid-liquid interface tension are considered to be linearly dependent on the temperature,

$\sigma_{i}=\sigma_{i 0}-\gamma_{i}\left(T_{i, I}-T_{0}\right)$.

The subscript $i=1,2$, respectively represents the liquid-liquid interface and the gas-liquid interface. $\sigma_{i 0}, i=1,2$, represents the surface tension at $T_{i, I}=T_{0} . T_{1, I}, T_{2, I}$, respectively, is the temperature at the liquid-liquid interface and gas-liquid interface. $\gamma_{i}, i=1,2$, represents the negative rate of surface tension with temperature. The values of the surface tension $\sigma_{i 0}$, and coefficients $\gamma_{i}$ are listed in Table 2. By using the depth and the physical properties of fluorinert FC70 as the non-dimensional scales, we introduced the depth ratio $h=\frac{h_{1}}{h_{2}}$, the density ratio $\rho=\frac{\rho_{1}}{\rho_{2}}$, the kinematical viscosity ratio $v=\frac{v_{1}}{v_{2}}$, the thermal diffusivity ratio $\kappa=\frac{\kappa_{1}}{\kappa_{2}}$, the thermal conductivity ratio $\chi=\frac{\chi_{1}}{\chi_{2}}$. We considered the fully three-dimensional system and scale all the distances on the depth of FC70. The velocities $\mathbf{v}=(u, v, w)$, pressure $p$, temperature differences $T-T_{0}$, and the time $t$ are refereed to scales $\frac{\kappa_{2}}{h_{2}}, \frac{\rho_{2} \kappa_{2}^{2}}{h_{2}^{2}}, b h_{2}, \frac{h_{2}^{2}}{\kappa_{2}}$ respectively. The following dimensionless groups are listed:

$\operatorname{Pr}=\frac{v_{2}}{\kappa_{2}}, \quad M a_{1}=\frac{\gamma_{1} b h_{2}^{2}}{\rho_{2} v_{2} \kappa_{2}}, \quad M a_{2}=\frac{\gamma_{2} b h_{2}^{2}}{\rho_{2} v_{2} \kappa_{2}}$,

where $M a_{2}, M a_{1}$ are Marangoni numbers, $\operatorname{Pr}$ is the Prandtl number.

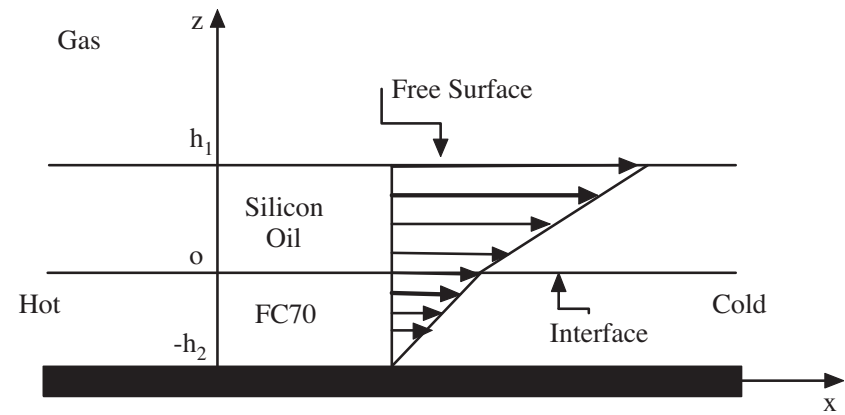

Fig. 2. A sketch of a geometry of the infinite, horizontal bilayer system. A constant temperature gradient imposed along the bilayer gives rise to the linear basic-state velocity profile. 
Table 1

Physical properties of Silicon oil 10cS and Fluorinert FC70.

\begin{tabular}{lll}
\hline & Silicon oil 10cs & Fluorinert FC70 \\
\hline Density $\left(\mathrm{kg} \mathrm{m}^{-3}\right)$ & $9.35 \times 10^{2}$ & $1.94 \times \times 10^{3}$ \\
Kinematical viscosity $\left(\mathrm{m}^{2} \mathrm{~s}^{-1}\right)$ & $1.00 \times 10^{-5}$ & $1.40 \times 10^{-5}$ \\
Thermal diffusivity $\left(\mathrm{m}^{2} \mathrm{~s}^{-1}\right)$ & $9.50 \times 10^{-8}$ & $3.40 \times 10^{-8}$ \\
Thermal conductivity $\left(\mathrm{J} \mathrm{m}^{-1} \mathrm{~s}^{-1} \mathrm{~K}^{-1}\right)$ & 0.134 & $6.99 \times 10^{-2}$ \\
\hline
\end{tabular}

Table 2

Physical properties of the surface tension.

\begin{tabular}{lll}
\hline & $\sigma_{0}\left(\mathrm{~N} \mathrm{~m}^{-1}\right)$ & $\gamma\left(\mathrm{N} \mathrm{m}^{-1} \mathrm{~K}^{-1}\right)$ \\
\hline Gas-Silicon oil 10cs interface & $20.1 \times 10^{-3}$ & $6.80 \times \times 10^{-5}$ \\
Silicon oil 10cs-Fluorinert FC70 interface & $7.60 \times 10^{-3}$ & $4.46 \times 10^{-5}$ \\
\hline
\end{tabular}

The dimensionless governing equations are,the continuity equation, the Navier-Stokes equations and the energy equation.

For the upper layer:

$\nabla \cdot \mathbf{v}_{1}=0$

$\frac{\partial \mathbf{v}_{1}}{\partial t}+\left(\mathbf{v}_{1} \cdot \nabla\right) \mathbf{v}_{1}=-\frac{\operatorname{Pr}}{\rho} \nabla p_{1}+v \operatorname{Pr} \triangle \mathbf{v}_{1}$

$\frac{\partial \theta_{1}}{\partial t}+\left(\mathbf{v}_{1} \cdot \nabla\right) \theta_{1}=\kappa \triangle \theta_{1}$.

For the lower layer:

$\nabla \cdot \mathbf{v}_{2}=0$

$\frac{\partial \mathbf{v}_{2}}{\partial t}+\left(\mathbf{v}_{2} \cdot \nabla\right) \mathbf{v}_{2}=-\operatorname{Pr} \nabla p_{2}+\operatorname{Pr} \triangle \mathbf{v}_{2}$

$\frac{\partial \theta_{2}}{\partial t}+\left(\mathbf{v}_{2} \cdot \nabla\right) \theta_{2}=\Delta \theta_{2}$

The subscript 1, 2 represents the upper layer and the lower layer respectively.

Prior to setting the boundary conditions at the two interfaces, the Crispation number $C r_{i}=\frac{\rho_{2} v_{2} \kappa_{2}}{\sigma_{i} h_{2}}$ is defined. It measures the magnitude of the effects of surface tension. Using the values in Tables 1 and 2, the order of $\mathrm{Cr}_{i}$, respectively, is about $O\left(10^{-5}\right)$, if the depth of the lower layer is about $10^{-2} \mathrm{~m}$. The small value of $C r_{i}$ indicates that the fluid system may justify the assumption of non-deformable interfaces that we made in Section 1.

Then, on the gas-liquid surface $z=h$, boundary conditions can be expressed as follows,

$w_{1}=0, \quad \rho v \partial_{z} u_{1}+M a_{2} \partial_{x} \theta_{1}=0, \quad \rho v \partial_{z} v_{1}+M a_{2} \partial_{y} \theta_{1}=0$,

$\partial_{z} \theta_{1}+B i\left(\theta_{1}-\theta_{\infty}\right)=0$.

$B i$ is the Biot number; it measures the efficiency of heat transfer at the surface. $\theta_{\infty}$ is the non-dimensional temperature of the bounding gas far from the gas-liquid interface, and is determined by the basic state solution under particular consideration which is not an independent parameter here.

On the liquid-liquid interface $z=0$, velocity, temperature and heat flux are assumed continuous,

$u_{2}=u_{1}, \quad v_{2}=v_{1}, \quad \theta_{2}=\theta_{1}, \quad \partial_{z} \theta_{2}=\chi \partial_{z} \theta_{1}$.

The stress balance conditions at $z=0$ are,

$\partial_{z} u_{2}-\rho v \partial_{z} u_{1}=-M a_{1} \partial_{x} \theta_{2}, \quad \partial_{z} v_{2}-\rho v \partial_{z} v_{1}=-M a_{1} \partial_{y} \theta_{2}$.

The non-deformable interface assumption gives the wall-normal velocity condition at $z=0$, $w_{2}=w_{1}=0$.

On the rigid lower plane $z=-1$, the non-slip boundary condition and adiabatic condition are considered,

$u_{2}=v_{2}=w_{2}=\partial_{z} \theta_{2}=0$.

\subsection{The base state}

We considered parallel-flow solutions of the system (3)-(14). The non-dimensional temperature at the gas-liquid interface $z=h$ is set to $\theta_{\text {int }}=-\chi$, and Eq. (10) is re-written as,

$\partial_{z} \theta_{1}+\operatorname{Bi}\left(\theta_{1}-\theta_{\text {int }}\right)+\operatorname{Bi}\left(\theta_{\text {int }}-\theta_{\infty}\right)=0$.

If let $Q=B i\left(\theta_{\text {int }}-\theta_{\infty}\right)$, we got the same form as described in Davis's paper [6], and $Q$ was defined as an imposed heat flux in [4,5]. Here, we assumed $\theta_{1}=\theta_{\text {int }}=-\chi$, which implied an constant heat flux $Q$ should be imposed by the particular consideration.

The parallel-flow solution was obtained by solving the governing Eqs. (3)-(14), and was defined as follows:

$$
\begin{aligned}
\overline{\mathbf{v}}_{\mathbf{1}}= & \left(\bar{u}_{1}, \bar{v}_{1}, \bar{w}_{1}\right)=\left(\frac{M a_{2}}{\rho v} z+M a_{1}+M a_{2}, 0,0\right), \\
\overline{\mathbf{v}}_{\mathbf{2}}= & \left(\bar{u}_{2}, \bar{v}_{2}, \bar{w}_{2}\right)=\left(\left(M a_{1}+M a_{2}\right) z+M a_{1}+M a_{2}, 0,0\right), \\
\bar{\theta}_{1}= & -\chi-\left(\frac{M a_{2} z^{3}}{6 \rho v \kappa}+\frac{\left(M a_{1}+M a_{2}\right) z^{2}}{2 \kappa}+\frac{\left(M a_{1}+M a_{2}\right) z}{2 \chi}\right) \\
& +\frac{M a_{2} h^{3}}{6 \kappa \rho v}+\frac{\left(M a_{1}+M a_{2}\right) h^{2}}{2 \kappa}+\frac{\left(M a_{1}+M a_{2}\right) h}{2 \chi}, \\
\bar{\theta}_{2}= & -\chi-\frac{M a_{1}+M a_{2}}{6}\left(z^{3}+3 z^{2}+3 z\right)+\frac{M a_{2} h^{3}}{6 \kappa \rho v} \\
& +\frac{\left(M a_{1}+M a_{2}\right) h^{2}}{2 \kappa}+\frac{\left(M a_{1}+M a_{2}\right) h}{2 \chi} .
\end{aligned}
$$

The base pressure in each layer was constant. The flow-induced temperature field made the bottom of the lower layer warmer than the liquid-liquid interface, and the liquid-liquid interface warmer than the gas-liquid surface.

\subsection{Linear stability theory}

We introduced infinitesimal disturbances to the system (3)(14) as follows,

$$
\begin{aligned}
& \left(\mathbf{v}_{1}, p 1, \theta_{1}\right)=\left(\overline{\mathbf{v}}_{1}, \bar{p}_{1}, \bar{\theta}_{1}\right)+\left(\mathbf{v}_{1}^{\prime}, p_{1}^{\prime}, \theta_{1}^{\prime}\right), \\
& \left(\mathbf{v}_{2}, p_{2}, \theta_{2}\right)=\left(\overline{\mathbf{v}}_{2}, \bar{p}_{2}, \bar{\theta}_{2}\right)+\left(\mathbf{v}_{2}^{\prime}, p_{2}^{\prime}, \theta_{2}^{\prime}\right) .
\end{aligned}
$$

Substituting Eqs. (20) and (21) into the governing system (3)-(14), and after linearizing, we obtained the linearized perturbed system.

For the upper layer:

$\nabla \cdot \mathbf{v}_{1}^{\prime}=0$

$\partial_{t} \mathbf{v}_{1}^{\prime}+\overline{\mathbf{v}}_{\mathbf{1}} \cdot \nabla \mathbf{v}_{1}^{\prime}+\mathbf{v}_{1}^{\prime} \cdot \nabla \overline{\mathbf{v}}_{1}=-\frac{\operatorname{Pr}}{\rho} \nabla p_{1}^{\prime}+v \operatorname{Pr} \triangle \mathbf{v}_{1}^{\prime}$,

$\partial_{t} \theta_{1}^{\prime}+\bar{u}_{1} \partial_{x} \theta_{1}^{\prime}+u_{1}^{\prime} \partial_{x} \bar{\theta}_{1}+w_{1}^{\prime} \partial_{z} \bar{\theta}_{1}=\kappa \Delta \theta_{1}^{\prime}$.

For the lower layer:

$\nabla \cdot \mathbf{v}_{2}^{\prime}=0$

$\partial_{t} \mathbf{v}_{2}^{\prime}+\overline{\mathbf{v}}_{\mathbf{2}} \cdot \nabla \mathbf{v}_{2}^{\prime}+\mathbf{v}_{2}^{\prime} \cdot \nabla \overline{\mathbf{v}}_{\mathbf{2}}=-\operatorname{Pr} \nabla p_{2}^{\prime}+\operatorname{Pr} \Delta \mathbf{v}_{2}^{\prime}$,

$\partial_{t} \theta_{2}^{\prime}+\bar{u}_{2} \partial_{x} \theta_{2}^{\prime}+u_{2}^{\prime} \partial_{x} \bar{\theta}_{2}+w_{2}^{\prime} \partial_{z} \bar{\theta}_{2}=\Delta \theta_{2}^{\prime}$. 
The perturbed boundary conditions take the same form as Eqs. (9)(14) except (10). We wrote the dimensionless Newton's cooling law for the perturbed system,

$\partial_{z} \theta_{1}^{\prime}+B i \theta_{1}^{\prime}=0$.

In this paper, we considered the normal mode analysis,

$\left[\mathbf{v}_{1}^{\prime}, p_{1}^{\prime}, \theta_{1}^{\prime}\right]=\left[\mathbf{V}_{1}, P_{1}, \Theta_{1}\right](z) e^{i(\alpha x+\beta y)+\lambda t}$,

$\left[\mathbf{v}_{2}^{\prime}, p_{2}^{\prime}, \theta_{2}^{\prime}\right]=\left[\mathbf{V}_{2}, P_{2}, \Theta_{2}\right](z) e^{i(\alpha x+\beta y)+\lambda t}$,

$\alpha$ and $\beta$ are streamwise disturbance wave-number and spanwise disturbance wave-number respectively. $\lambda$ is the complex eigenvalue $\lambda=\lambda_{r}+i \lambda_{i}$ whose real part $\lambda_{r}$ is defined as the effective temporal growth rate. A overall wave-number $k=\sqrt{\alpha^{2}+\beta^{2}}$, and the phase speed $c=-\frac{\lambda_{i}}{k}$ are defined here. The Chebyshev collocation numerical method $[19,20]$ is used to resolve the eigenvalue problem in $\lambda$ of the linearized system.

\section{Results of $B i=0$}

In this section, we discussed the quasi-adiabatic condition at the gas-liquid surface, i.e. $B i \rightarrow 0$. $B i$ cannot be zero, since the ambient temperature $\theta_{\infty}$ is proportional to $\frac{1}{B i}$ under the particular consideration. However, to the perturbed system, we can let $B i=0$ to study the influence of the depth ratio on the nature of stability of this system. $B i=0$ was also proposed in the works of Smith and Davis [4] and Davis [6]. System (22)-(27) together with its boundary conditions must be solved numerically to characterize completely the three-dimensional instability of the basic state, because a Squire's theorem for this flow is not possible. First, we restricted ourselves here to the two typical cases of $\alpha \equiv 0$ and $\beta \equiv 0$.
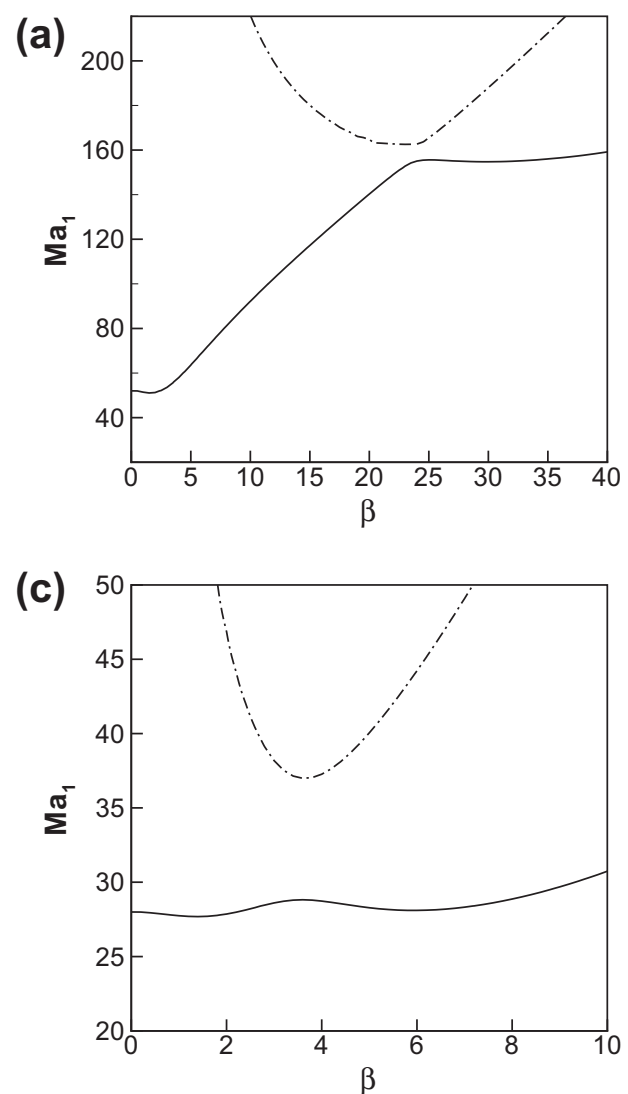

\section{1. $\alpha=0$}

When $\alpha=0$, the linearized equations are decoupled. We introduced a stream function here,

$v^{\prime}=\partial_{z} \phi, \quad w^{\prime}=-\partial_{y} \phi$

and wrote the normal mode of stream function as $\phi$ $(y, z, t)=\Phi(z) e^{i \beta y+\lambda t}$. In this paper, $\Phi=\Phi_{R}+i \Phi_{I}$, and $\Theta=\Theta_{R}+i \Theta_{I}$, and the two eigenfunctions are rescaled by the maximum of $\|\Phi\|=\sqrt{\Phi_{R}^{2}+\Phi_{I}^{2}}$ and $\|\Theta\|=\sqrt{\Theta_{R}^{2}+\Theta_{I}^{2}}$ respectively. That is $\Phi \rightarrow \frac{\Phi}{\|\Phi\|}$, and $\Theta \rightarrow \frac{\Theta}{\|\Theta\|}$.

In Fig. 3, marginal curves of four typical cases are shown. In Figs. 4(a), and 5(a), the eigenfunctions of temperature almost do not change in the upper layer, and the eigenfunctions of the stream function indicate that convection of the bilayer is co-rotating. This indicates unstable mode in the system is the thermal-coupling mode. While in Fig. 5(b), the profile of $\Phi$ indicates that the convection in the lower layer is very weak, and this is the weakly decoupled mode. The two coupling modes in Fig. 5 are stationary modes. A special example $h=0.25$ is presented here. The marginal curve in Fig. 3(c) presents a bi-modal structure which indicates that convection in the system may be initiated in two different coupling modes. When $\beta=1.5$, it is thermal-coupling mode as shown in Fig. 5(a); and when $\beta=6$, convection is in the form of weakly decoupled mode as shown in Fig. 5(b).

In Fig. 3, when the Marangoni number $M a_{1}$ exceeds some critical value, there are two temporal growth rates $\lambda_{r}>0$. We denote the larger one $\lambda_{r}=\lambda_{1}$ as the first eigenvalue, and the other $\lambda_{r}=\lambda_{2}$ as the second eigenvalue. The mode corresponding to $\lambda_{1}$ is defined as the first mode, and the mode corresponding to $\lambda_{2}$ as the second mode. When $\lambda_{1}>\lambda_{2}$, the second mode is suppressed by the first

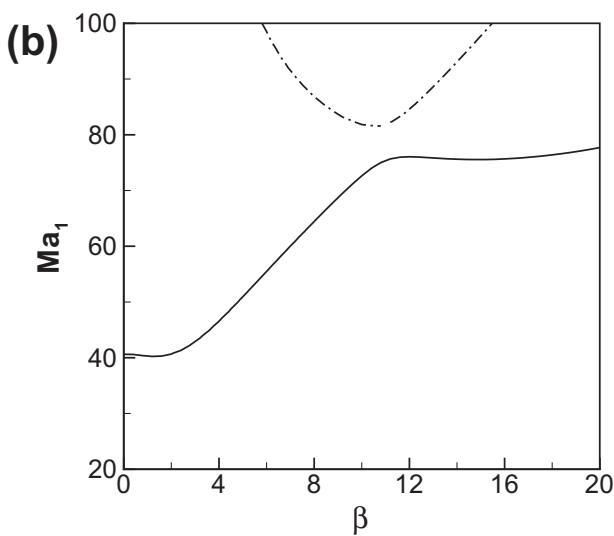

(d)

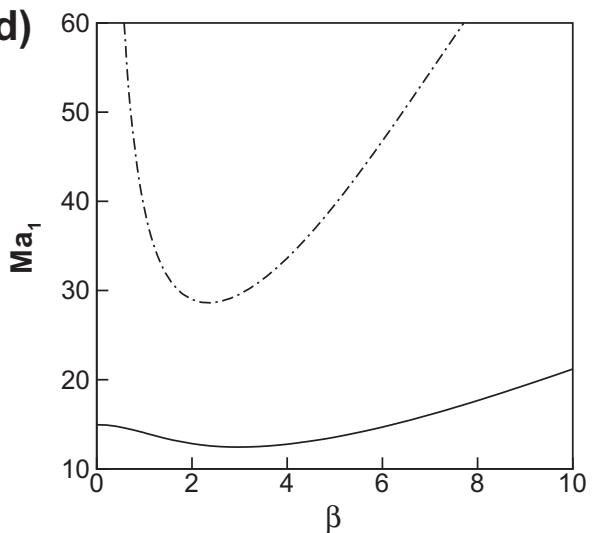

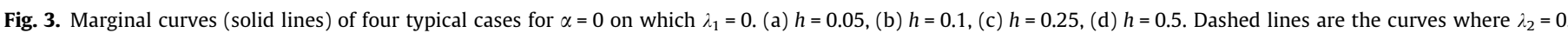



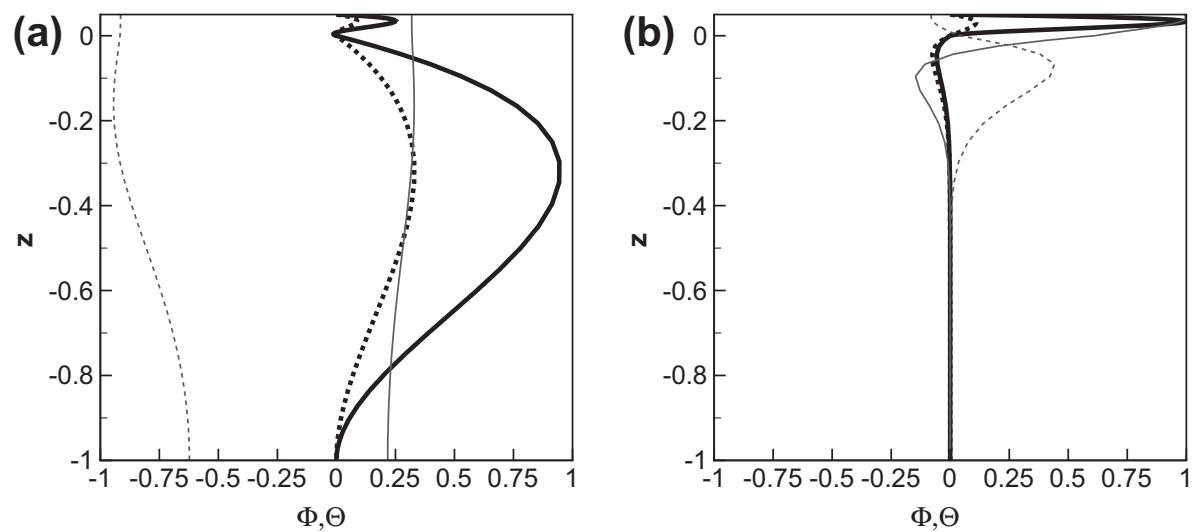

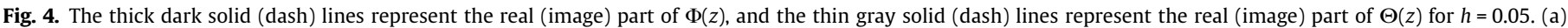
thermal-coupling mode, $M a_{1}=50.5, \beta=1.6, B i=0$; (b) oscillatory mode, $M a_{1}=170, \beta=20$.
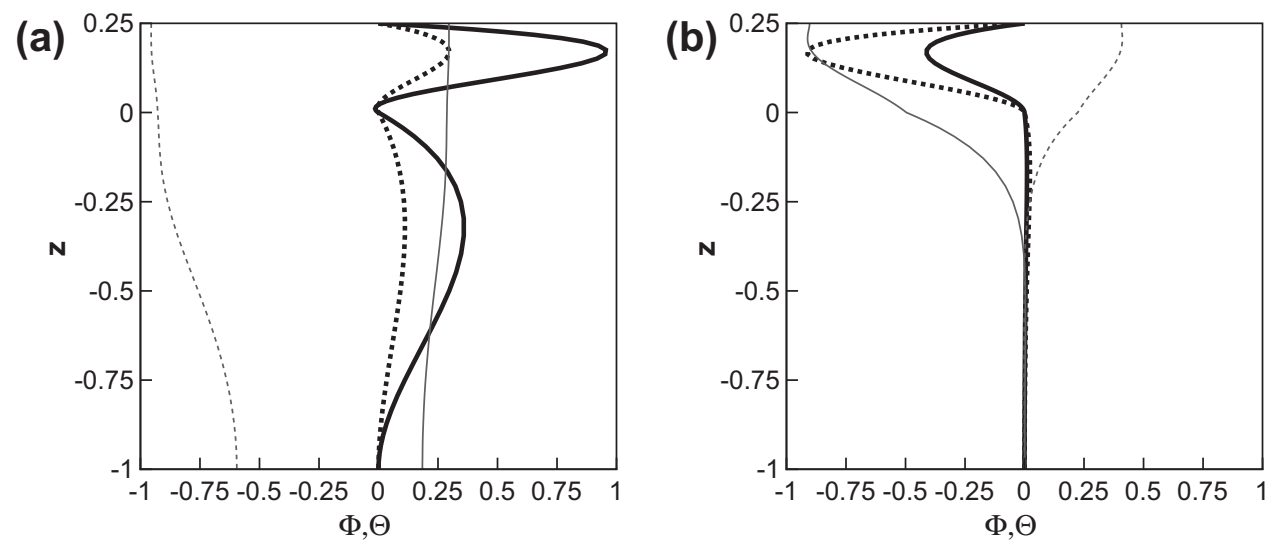

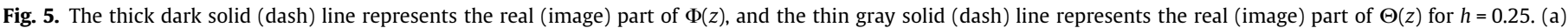
Thermal-coupling, $M a_{1}=27.8, \beta=1.5$; (b) Weakly decoupled, $M a_{1}=28.4, \beta=6$.
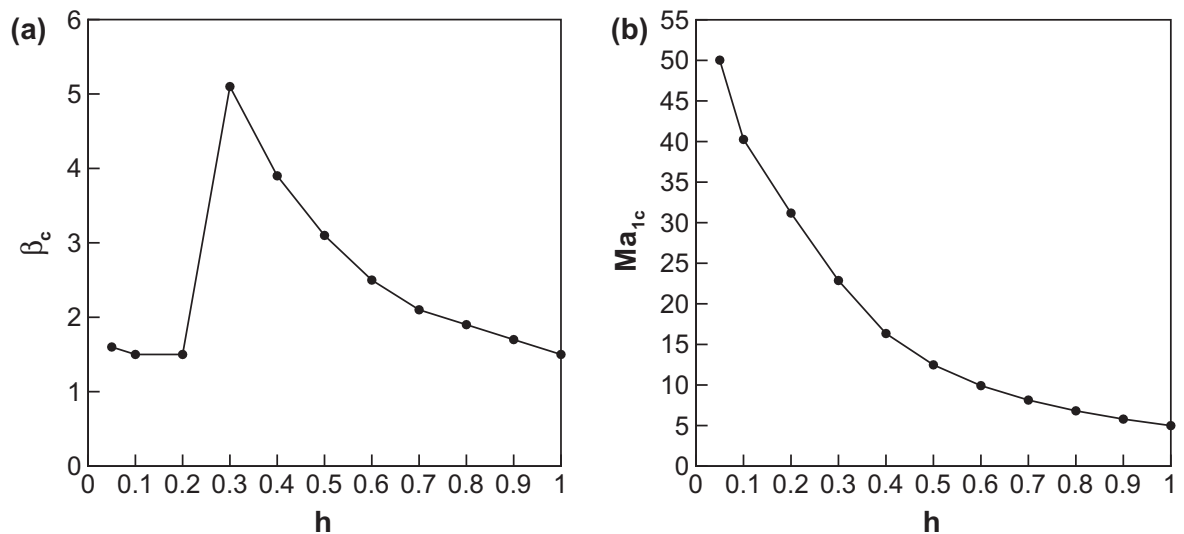

Fig. 6. (a) The critical wave number $\beta_{c}$ versus the depth ratio $h$; (b) The critical Marangoni number $M a_{1 c}$ versus depth ratios $h$.

mode, as a result, the convection type is in the form of stationary mode. When $\lambda_{1}=\lambda_{2}$, the oscillatory mode occurs. It is found that, when the depth is small $(h<0.3)$, and for certain pair of $\left(M a_{1}, \beta\right)$, oscillatory mode exists. In Fig. 4(b), we observe that the unstable mode is oscillating between thermal-coupling mode and weakly decoupled mode. This oscillatory mode takes the form of traveling waves propagating in either spanwise direction. When the depth ratio $h>0.3$, the oscillatory modes are not observed, because convection in the upper layer always dominates.
Fig. 6 presents the critical wave number $\beta_{c}$ and Marangoni number $M a_{1 c}$ versus the depth ratio $h$. In Fig. 6(a), it is obvious that there is a jump at the depth ratio of $h=0.3$, which indicates, for critical instability, the type of convection changes with the depth ratio $h$. A further analysis is shown here. Pearson [3] indicated that the scales of Marongoni convection cells are dependent on the depth of the liquid layer. In this case, when $h<0.3$, bearing in mind of the lower layer's dimensionless length, the critical wave number $\beta_{c} \approx 1.5$, which indicates that convection in lower layer dominates. 

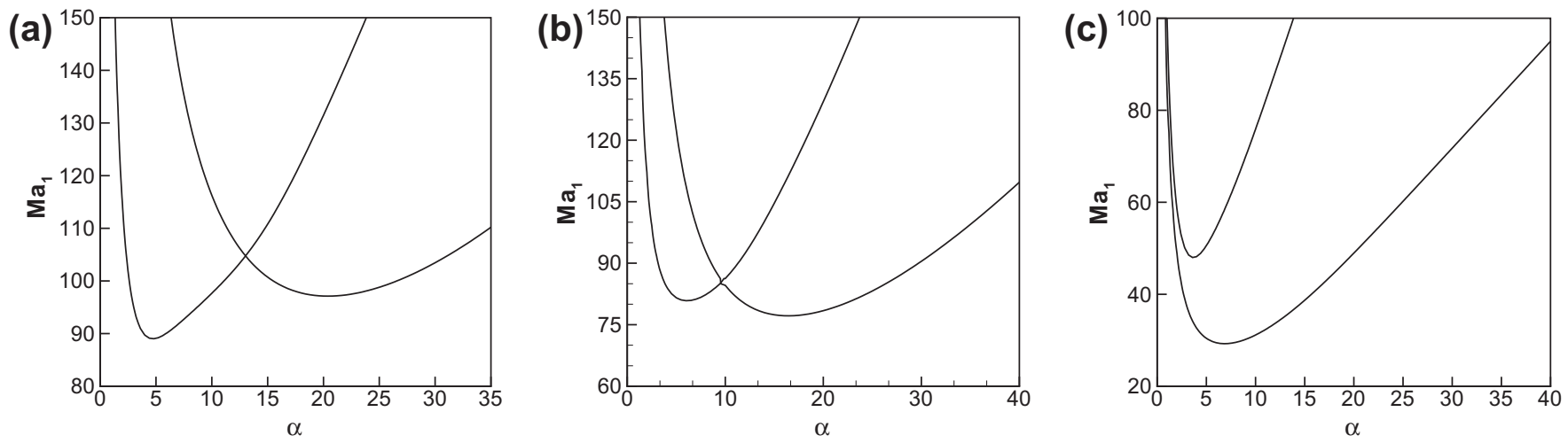

Fig. 7. Marginal curves of three typical cases at different depth ratios for $\beta=0$. (a) $h=0.08$, (b) $h=0.1$, (c) $h=0.25$.
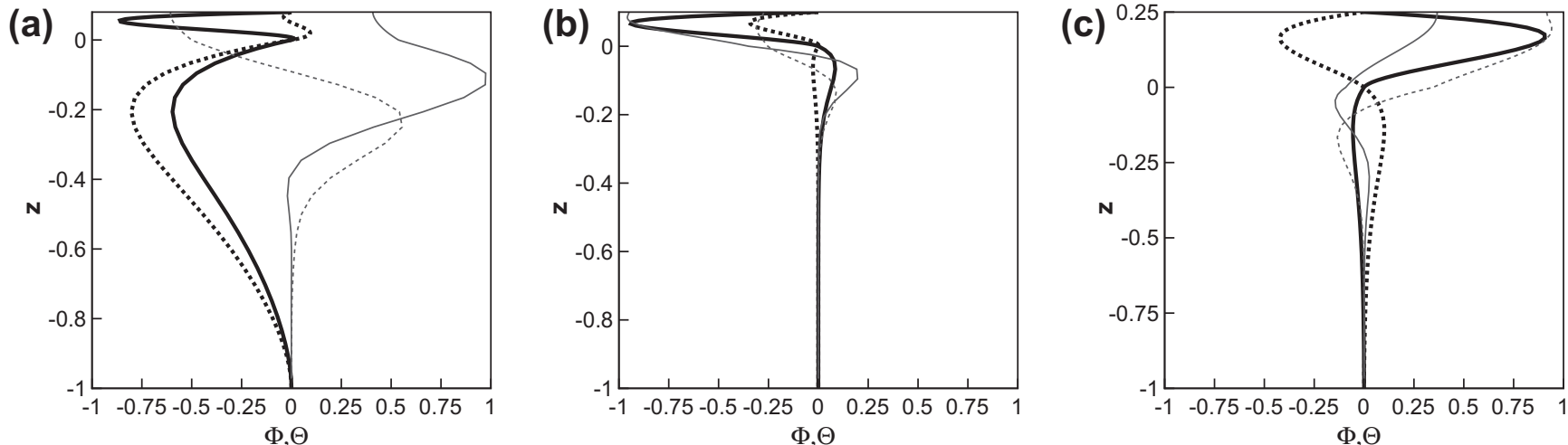

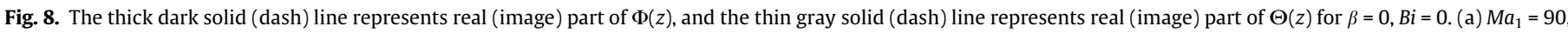
$\alpha=4.8, h=0.08$; (b) $M a_{1}=78, \alpha=16, h=0.1$; (c) $M a_{1}=30, \alpha=7, h=0.25$.
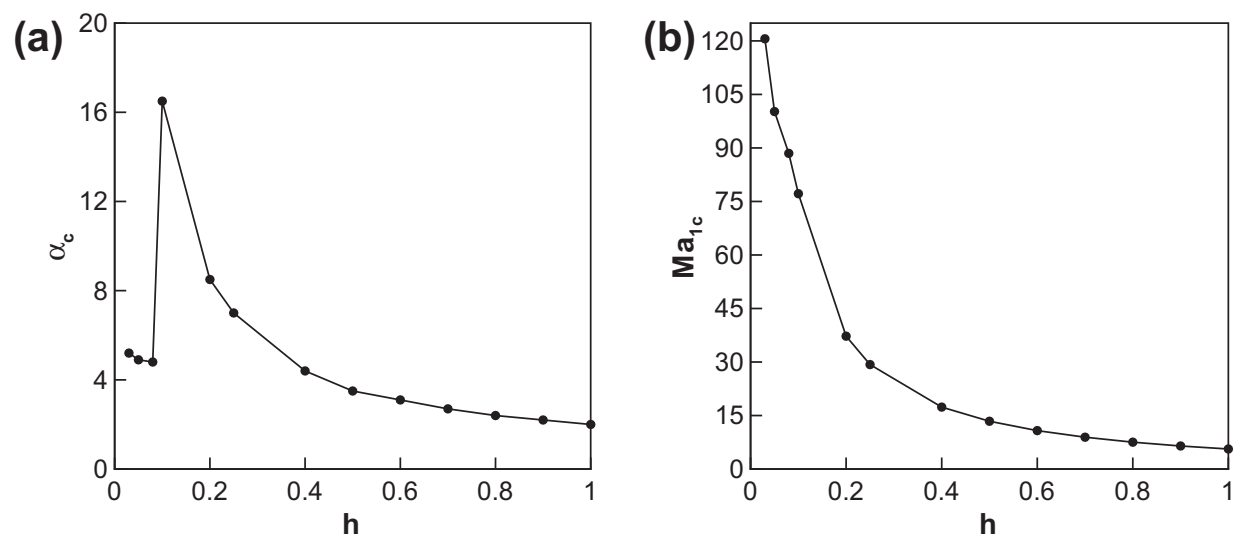

Fig. 9. (a) The critical wave number $\alpha_{c}$ versus the depth ratio $h$ for $\beta=0$; (b) The critical Marangoni number $M a_{1 c}$ versus the depth ratio $h$ for $\beta=0$.

When $h>0.3$, if we re-scale the all the lengths in the system by using the upper layer's depth as the length scale, then we obtain $\beta^{\prime}=\beta h$. Note that $\beta^{\prime} \approx 1.5$, which implies that the convection in the upper layer dominates. In Fig. 6(b), the critical Marangoni number $M a_{1 c}$ decreases with depth ratio $h$. In order to understand the reason, we refer to the heat flux boundary condition Eq. (28), and write it approximately as

$\partial_{z} \theta_{2}^{\prime} \approx \chi \frac{\left.\theta^{\prime}\right|_{z=0}-\theta_{\text {int }}^{\prime}}{h}$.

We assume that there is a hot spot on the liquid-liquid interface due to disturbances. Eq. (32) indicates that the hot spot is cooled through heat conduction in the upper layer. The heat conduction effects would be reduced with increasing the depth ratio $h$. Thus, the system becomes more unstable. As a result, the critical Marangoni number $M a_{1 c}$ decreases with increasing $h$.

\section{2. $\beta=0$}

We further investigated the spanwise homogeneous disturbances case in this section, i.e. $\beta=0$. In Fig. 7, marginal curves for three typical depth ratios are shown. Comparing Fig. 3 with Fig. 7, the system is more unstable to spanwise disturbances. 


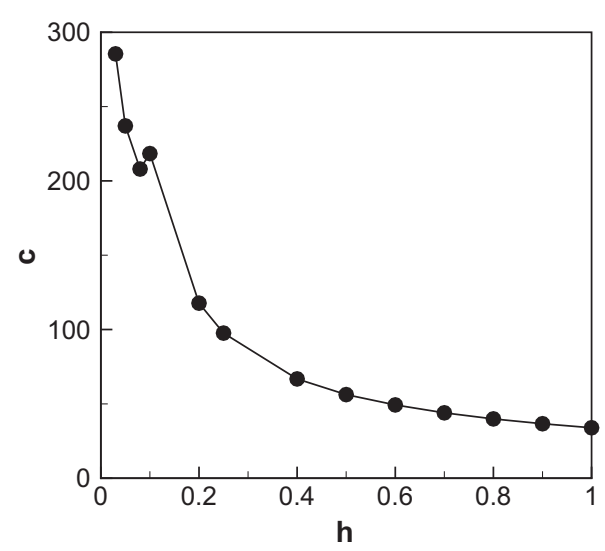

Fig. 10. The critical phase speed $c$ versus the depth ratio $h$ for spanwise homogeneous disturbances case.

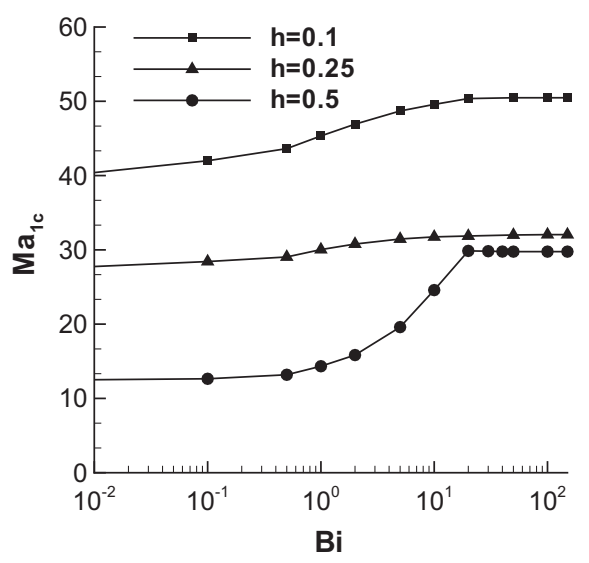

Fig. 11. The critical Marangoni number $M a_{1 c}$ versus $B i$ at different depth ratio for $\alpha=0$.

Here, the stream function is defined as follows:

$u^{\prime}=\partial_{z} \phi, \quad w^{\prime}=-\partial_{x} \phi$

and, the normal mode of the stream function is $\phi(x, z, t)=\Phi(z) e^{i \alpha x+\lambda t}$. Fig. 8 shows the eigenfunctions $\Phi$ and $\Theta$. The eigenfunctions are also re-scaled as we made in the previous section. It is obvious, convection in the bilayer system is in the form of oscillatory mode as shown in Fig. 8.
In Figs. 9(a) and 10, there is a jump near $h=0.1$ respectively. When $h<0.1$, convection in the lower layer dominates, while convection in the upper layer dominates when $h>0.1$. Fig. 9(b) shows that the critical Marangoni number $M a_{1 c}$ decreases with increasing depth ratio $h$. The physical reason is similar to that we discussed in the previous section. In Fig. 10, the critical phase speed $c$ versus the depth ratio is shown, and from which we obtain the perturbed flow travels in the same direction as base flow.

\section{Results of varying $B i$}

\section{1. $\alpha=0$}

This section discussed the influences of $B i$ on stability of the system, and the streamwise homogeneous disturbances case was studied. When $B i \rightarrow 0$, convection in the system is initiated in the form of weakly decoupled mode when $h>0.3$. However, the weakly decoupled mode can be weakened by the cooling of the bounding gas when $B i>0$. If $B i$ is sufficiently large, convection would be driven by the liquid-liquid interface tension rather than by the liquid-liquid interface tension, and be initiated in the form of mechanical-coupling mode. Fig. 11 shows the critical Marangoni number $M a_{1 c}$ versus $B i$ which provides a first sight of the type of convection in the system. It is obvious, for $h=0.1,0.25$, the critical Marangoni number $M a_{1 c}$ increases with increasing $B i$, which implies that convection in the lower layer always dominates and increasing $B i$ enhance the stability of the system. While $h=0.5$, when the $B i$ exceeds a threshold value, we observe $M a_{1 c}$ decreases with increasing $B i$ slightly, which implies that the domination of convection shifts from the upper layer to the lower layer.

For a certain $B i$ and some values of $M a_{1}$ and $\beta$, convection may oscillate between the mechanical coupling mode and the thermal coupling mode. First, we showed that for some certain large $B i$, convection in the system could be initiated in the form of mechanical coupling mode. To illustrate this, the eigenfunction $\Phi(z)$ for the critical unstable case is plotted in Fig. 12 by fixing the depth ratio $h=0.25$ to see the evolution of the convection mode versus $B i$. Clear, we can observe the mechanical coupling mode in Fig. 12(c).

The marginal curves of depth ratio $h=0.5$ for different $B i$ are shown in Fig. 13. The dashed lines in Fig. 13 represent the oscillatory branches. What is interesting that there exists a stable area in the $M a_{1}-\beta$ plane as shown Fig. 13(b). The gas-liquid interface tension acts an important role, and the mechanism can be understood as follows. Suppose that convection is driven by liquid-liquid interface tension, and is initiated in the form of mechanical coupling mode. When $M a_{1}$ increases, the thermal capillary force of the gas-liquid interface increases, which suppresses the
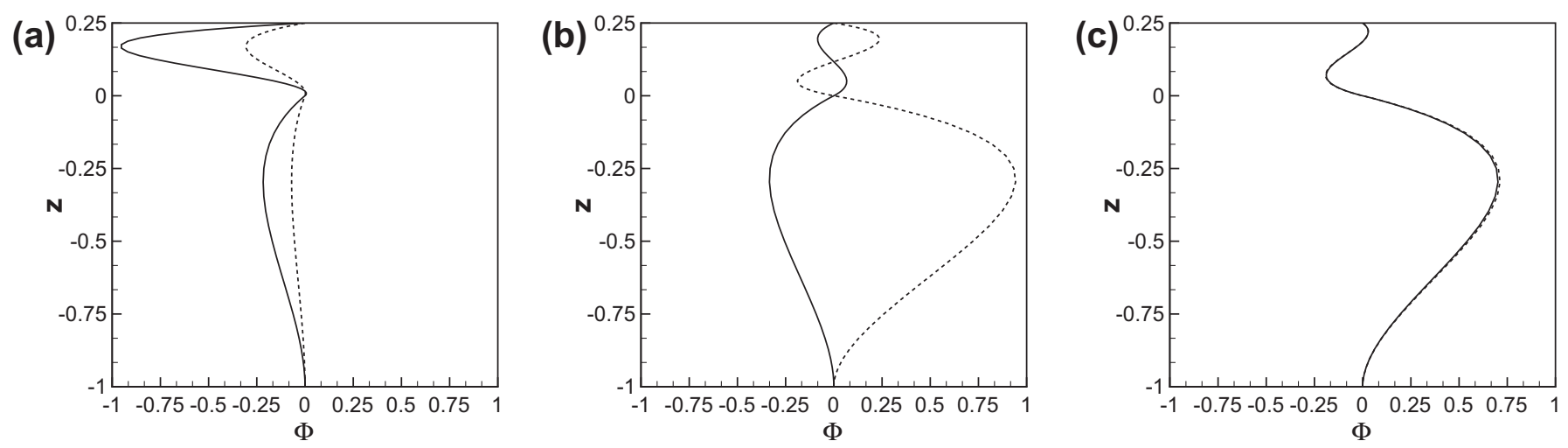

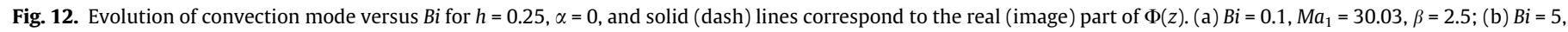
$M a_{1}=31.46, \beta=2.8 ;$ (c) $B i=10, M a_{1}=31.74, \beta=2.7$. 

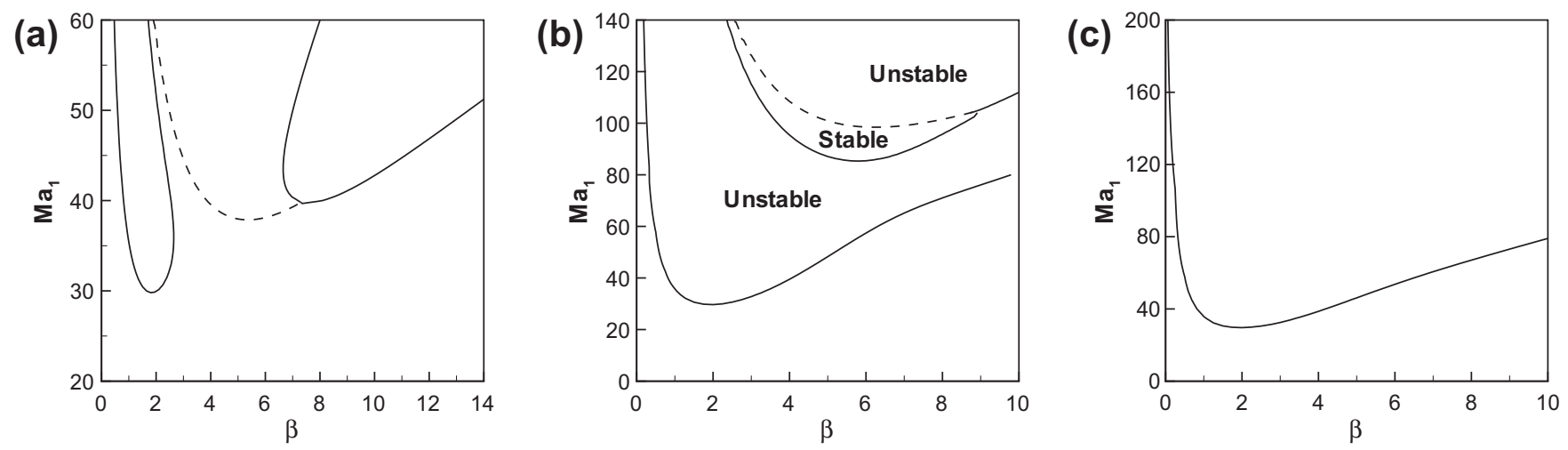

Fig. 13. Marginal curves for $h=0.5$. Dash lines correspond to the oscillatory branch, and solid lines correspond to stationary branch. (a) $B i=30$; $(b) B i=300$; $(c) B i=\infty$.
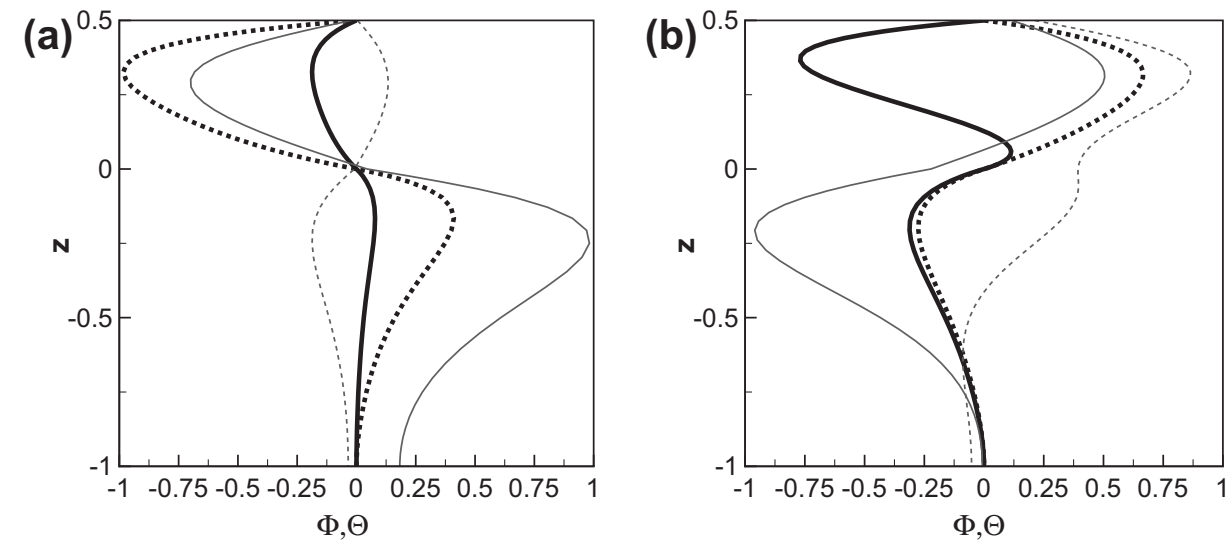

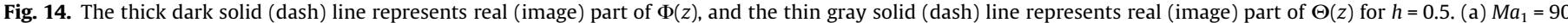
$\beta=6 B i=300$, convection in both layer vanishes when time $t \rightarrow \infty ;(b) M a_{1}=38, \beta=5, B i=30$.

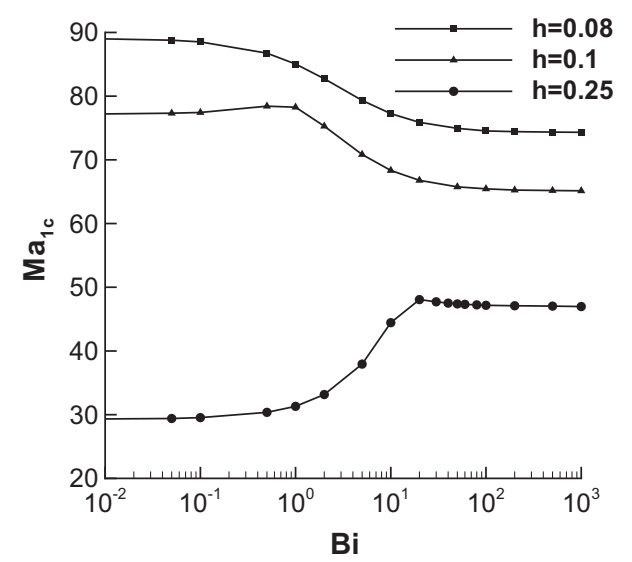

Fig. 15. The critical Marangoni number $M a_{1 c}$ versus $B i$ at different depth ratio.

convection in the upper layer. Two possible results may happen. One is that, because of the suppression of the upper layer, the system may be stable, and the other is the generation of oscillatory mode. Two special cases are presented here. Eigenfunction $\Phi$ in Fig. 14(a) suggests that convection in the upper layer is stronger than the lower layer. However, the distribution of $\Theta$ implies that disturbances decay with time. In Fig. 14(b), the eigenfunction $\Phi$ indicates that convection in the bilayer system takes the form of oscillatory mode which is in the cyclic variation between thermal coupling mode and mechanical-coupling mode. The oscillatory mode takes the form of traveling wave propagating in either spanwise direction.

\section{2. $\beta=0$}

The influence of $B i$ on the spanwise homogeneous disturbance case is discussed in this subsection. The critical Marangoni number $M a_{1 c}$ versus $B i$ is plotted in Fig. 15 which reflects the influence of $B i$ on the stability of the system. When $h=0.08$, convection in the lower layer dominates, and in Fig. 15, the critical Marangoni number $M a_{1 c}$ decreases with $B i$, which indicates the upper layer suppresses convection in the lower layer. When $B i \rightarrow \infty$, the critical Marangoni number $M a_{1 c} \rightarrow 74.31$. But, when $h=0.1$, or $h=0.25$, convection in the upper layer dominates when $B i$ is less than some critical value. It is clear that, when $B i$ is less than the critical value, a larger $B i$ will lead the system to be more stable due to the cooling of the bounding gas. When $B i$ exceeds the critical value, convection in the lower layer dominates, as a result, the critical Marangoni number decreases with increasing Bi. In Fig. 15, when $h=0.1$, $B i \rightarrow \infty$, the critical Marangoni number $M a_{1 c} \rightarrow 65.10$; and when $h=0.25, B i \rightarrow \infty$, the critical Marangoni number $M a_{1 c} \rightarrow 46.97$.

\section{Three dimensional disturbances: $\alpha \neq \mathbf{0}$ and $\boldsymbol{\beta} \neq \mathbf{0}$}

This section investigates the three dimensional disturbances on the stability of the bilayer system, because the Squire's theorem for this flow is not possible as mentioned in Section 3. The marginal surface is defined as follows, 

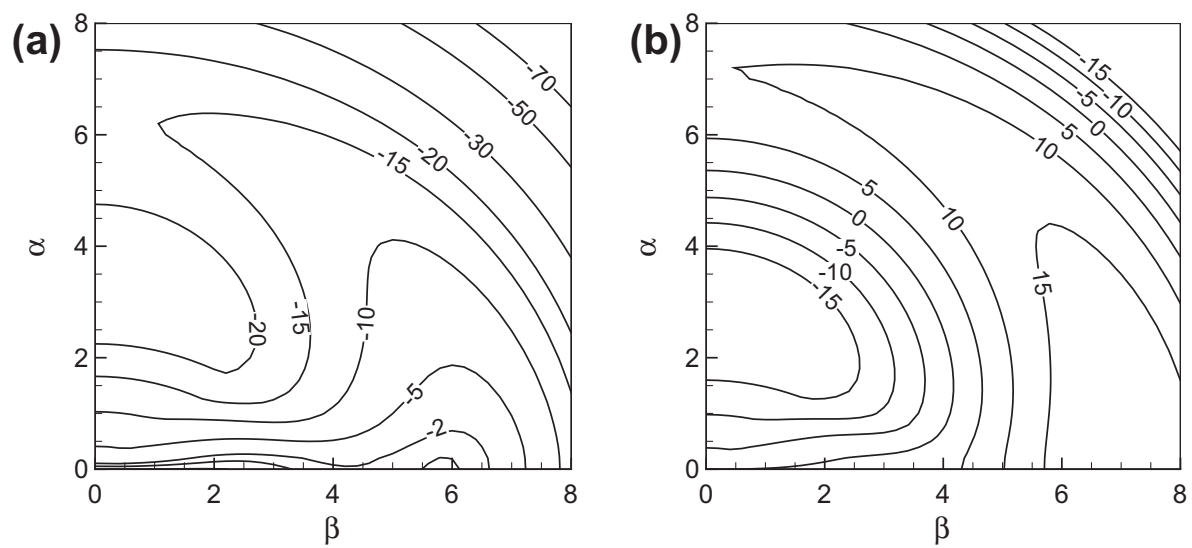

Fig. 16. Temporal grow rate on the $\alpha-\beta$ plane for $B i=0, h=0.25$. (a) $M a=28$; (b) $M a=30$.
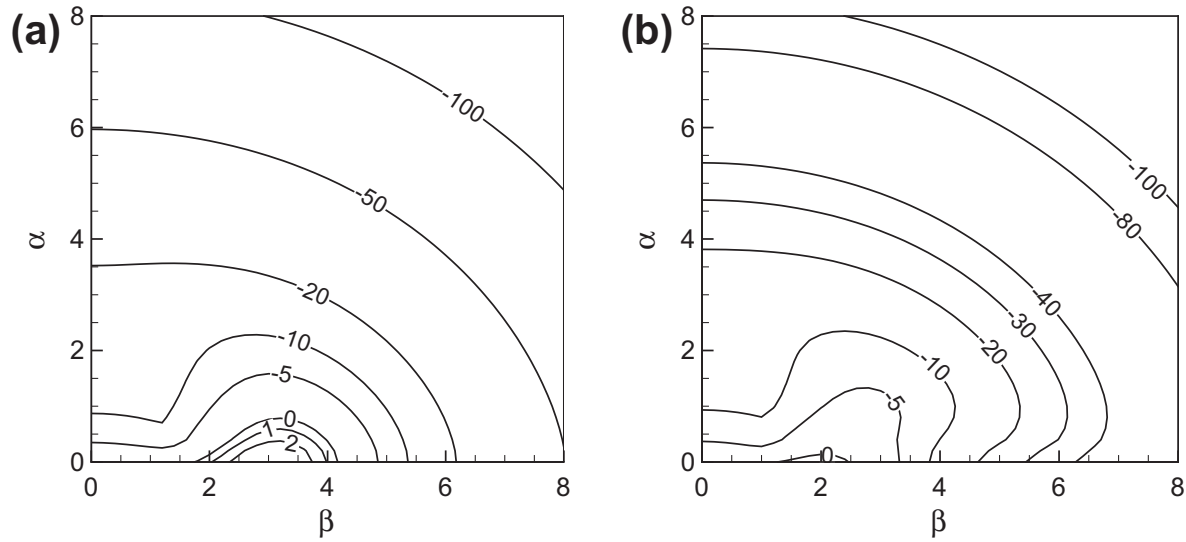

Fig. 17. Temporal grow rate on the $\alpha-\beta$ plane for $B i=30$. (a) $M a=35$ and $h=0.25$; (b) $M a=30, h=0.5$.

$M a_{1}=M a_{1}(\alpha, \beta, B i, h)$.

There must exist a minimum $M_{g}$ defined as the global critical Marangoni number on the marginal surface, i.e. when $M a_{1}<M_{g}$, disturbances decay to zero when time tends to infinity. We assume that the most preferred unstable mode travels in the direction,

$\vartheta=\tan ^{-1} \frac{\beta}{\alpha}$.

However, calculation of this marginal surface costs too much time. In another way, the temporal growth rate in the $\alpha-\beta$ plane is plotted by fixing $M a, B i$ and $h$. Fig. 16 shows the temporal growth rate for $B i=0$. In Fig. 16(a), we notice that the maximum temporal growth rate occurs at $\alpha=0$, which implies that spanwise disturbance makes the system be more unstable. In Fig. 16(b), the Marangoni number exceeds the critical Marangoni number $M_{g}$, and the maximum temporal growth rate also occurs at $\alpha=0$. Fig. 17, the temporal growth rate is plotted by fixing $B i=30$. In Fig. 17, the maximum growth rate occurs at $\alpha=0$ indicating spanwise disturbances are the major cause of destabilization in the system. Thus, the traveling direction of the most preferred unstable mode is $\vartheta=90^{\circ}$.

For the spanwise homogeneous disturbance, suppose there exists a hot spot on either liquid-liquid interface or gas-liquid interface. The hot spot flows to the downstream due to the base flow and is cooled by the downstream liquids. As a result, the disturbance is weakened, which indicates the base flow is stabilizing. However, disturbances in spanwise direction could not be weak- ened by the base flow. Therefore, the streamwise homogeneous disturbances are the most destabilizing factors in the system.

\section{Conclusion}

In this paper, we investigated the thermocapillary instabilities in a bilayer system consisting of silicon oil 10cS and fluorinert FC70 subjected to a constant horizontal temperature gradient. Either variation of gas-liquid interface tension or liquid-liquid interface tension can initiate convection in the system. Linear stability analysis was performed to study the complex coupling modes of convection in the system.

We studied two typical cases including the streamwise homogeneous disturbances and spanwise homogeneous disturbances in this paper. The influences of depth ratio and Biot number on the nature of convection were discussed. For the streamwise homogeneous disturbances case, convection may occur in the form of the thermal-coupling mode, or weakly decoupled mode when $B i=0$. When $B i>0$, convection may occur in the form of mechanical coupling mode. We found that the thermal-coupling mode interacts with the mechanical-coupling mode generating oscillatory mode which propagates in either spanwise direction. For the spanwise homogeneous disturbances case, it was found that convection always takes the form of traveling wave propagating in the same direction as the base flow. The three dimensional analysis showed that disturbances in spanwise direction were the major cause of destabilization in the system. 


\section{Acknowledgments}

The authors thank for the National Natural Science Foundation of China (Grant Nos. 50890182 and 11072249) and the Knowledge Innovation Project of Chinese Academy of Sciences. The author Ding Zijing thanks Dr. Liu Rong and Mr. Sabnavis Bindu Madhav for their several helpful discussions.

\section{References}

[1] P. Colinet, J.C. Legros, M.G. Velarde, Nonlinear Dynamics of Surface-TensionDriven Instabilities, Wiley-VCH, Berlin, 2001.

[2] D.D. Joseph, Y.Y. Renardy, Fundamentals of Two-Fluid Dynamics, SpringerVerlag, New York, 1993.

[3] J.R.A. Pearson, J. Fluid Mech. 4 (1958) 489.
[4] M. Smith, S.H. Davis, J. Fluid Mech. 132 (1983) 119.

[5] M. Smith, S.H. Davis, J. Fluid Mech. 132 (1983) 145.

[6] S.H. Davis, Annu. Rev. Fluid Mech. 19 (1987) 403.

[7] A.K. Sen, S.H. Davis, J. Fluid Mech. 121 (1982) 163.

[8] R.W. Zeren, W.C. Reynolds, J. Fluid Mech. 53 (1972) 305.

[9] S. Wahal, A. Bose, Phys. Fluids 31 (1988) 3502.

[10] S. Rasenat, F.H. Buss, I. Reherg, J. Fluid Mech. 199 (1989) 519

[11] Q.S. Liu, B. Roux, Int. J. Heat Mass Transfer 41 (1998) 11.

[12] Q.S. Liu, B.H. Zhou, N.T. Henri, W.R. Hu, Chin. Phys. Lett. 21 (2004) 4.

[13] Y.R. Li et al., Int. J. Heat Mass Transfer 52 (2009) 4769.

[14] I. Simanovskii, J. Mech. B 26 (2007) 422.

[15] I. Simanovskii et al., Phys. Fluids 21 (2009) 052106.

[16] A. Nepomnyashchy, I. Simanovskii, Phys. Fluids 19 (2007) 122103.

[17] A. Nepomnyashchy, I. Simanovskii, J. Fluid Mech. 661 (2010) 1.

[18] A. Nepomnyashchy, I. Simanovskii, Phys. Fluids 24 (2012) 032101.

[19] S.A. Orszag, J. Fluid Mech. 50 (1971) 689.

[20] C. Canuto, M.Y. Hussaini, A. Quarteroni, T.A. Zang, Spectral Methods in Fluid Dynamics, Springer-Verlag, Berlin, 1988. 